\title{
ON RINGS WHOSE RIGHT ANNIHILATORS ARE BOUNDED
}

\author{
SEO UN HWANG \\ Department of Mathematics, Busan National University, Busan 609-735, Korea \\ e-mail: hwangseo@dreamwiz.com \\ NAM KYUN KIM \\ College of Liberal Arts, Hanbat National University, Daejeon 305-719, Korea \\ e-mail:nkkim@hanbat.ac.kr \\ and YANG LEE \\ Department of Mathematics Education, Busan National University, Pusan 609-735, Korea \\ e-mail:ylee@pusan.ac.kr
}

(Received 27 August 2008; accepted 8 January 2009)

\begin{abstract}
Jacobson said a right ideal would be called bounded if it contained a non-zero ideal, and Faith said a ring would be called strongly right bounded if every non-zero right ideal were bounded. In this paper we introduce a condition that is a generalisation of strongly bounded rings and insertion-of-factors-property (IFP) rings, calling a ring strongly right $A B$ if every non-zero right annihilator is bounded. We first observe the structure of strongly right $\mathrm{AB}$ rings by analysing minimal non-commutative strongly right $\mathrm{AB}$ rings up to isomorphism. We study properties of strongly right $\mathrm{AB}$ rings, finding conditions for strongly right $\mathrm{AB}$ rings to be reduced or strongly right bounded. Relating to Ramamurthi's question (i.e. Are right and left SF rings von Neumann regular?), we show that a ring is strongly regular if and only if it is strongly right $\mathrm{AB}$ and right $\mathrm{SF}$, from which we may generalise several known results. We also construct more examples of strongly right $\mathrm{AB}$ rings and counterexamples to several naturally raised situations in the process.
\end{abstract}

2000 Mathematics Subject Classification. 16D25, 16D40, 16E50.

1. Introduction. Throughout this paper all rings are associative with identity unless otherwise stated, and all modules are unitary. Let $R$ be a ring. The prime radical, the set of all nilpotent elements and the Jacobson radical of $R$ are denoted by $P(R), N(R)$ and $J(R)$, respectively. For any non-empty subset $X$ of $R, r_{R}(X)$ and $\ell_{R}(X)$ denote the right annihilator and the left annihilator of $X$ in $R$, respectively. If $X$ is singleton, say $X=\{a\}$, then we write $r_{R}(a)\left(\ell_{R}(a)\right)$ in place of $r_{R}(\{a\})\left(\ell_{R}(\{a\})\right)$. We use $\operatorname{Mat}_{n}(R)$ to denote the $n \times n$ matrix ring over $R$. An element $c$ of $R$ is called right regular if $r_{R}(c)=0$, left regular if $\ell_{R}(c)=0$ and regular if $r_{R}(c)=0=\ell_{R}(c)$. A zero divisor means an element whose left and right annihilators are both non-zero. A ring is called a domain if every non-zero element is regular. It is well known that for a division ring $D$, a matrix in $\operatorname{Mat}_{n}(D)$ is regular if and only if it is invertible.

The notion of bounding a one-sided ideal by a two-sided ideal goes back at least to Jacobson [21]. He said that a right ideal of $R$ is bounded if it contains a non-zero ideal of $R$. This concept has been extended in several ways. From Faith [13], a ring $R$ is called strongly right (resp. left) bounded if every non-zero right (resp. left) ideal of $R$ 
contains a non-zero ideal. A ring is called strongly bounded if it is both strongly right and strongly left bounded. The class of strongly one-sided bounded rings has been observed by many authors [e.g. 7, 21, 34, 35, 37].

Due to Bell [6], a right (or left) ideal $I$ of a ring $R$ is said to have the insertion-offactors-property (simply, IFP) if $a b \in I$ implies $a R b \subseteq I$ for $a, b \in R$. So we shall call a ring $R$ an IFP ring if the zero ideal of $R$ has the IFP. Shin [32] used the term $S I$ for the IFP, while Narbonne [11] called IFP rings semi-commutative. A ring is called reduced if it has no non-zero nilpotent elements. A ring is called abelian if each idempotent is central. Simple computations give that reduced rings are IFP, and IFP rings are abelian. Note that a ring $R$ is IFP if and only if any right annihilator is an ideal if and only if any left annihilator is an ideal if and only if $a b=0$ implies $a R b=0$ for $a, b \in R$ [32, Lemma 1.2].

Due to Feller [14], a ring is right (resp. left) duo if every right (resp. left) ideal is an ideal. Right (resp. left) duo rings are both strongly right (resp. left) bounded and IFP. A ring is called duo if it is both right and left duo. Birkenmeier and Tucci [7, Proposition 6] showed that a ring $R$ is right duo if and only if $R / T$ is strongly right bounded for all ideals $T$ of $R$. For IFP rings we have a similar result in the following.

REMARK. For a ring $R$ the following conditions are equivalent:

(1) $R$ is IFP;

(2) for any right annihilator $A$ and any ideal $I$ in $R$ with $I \subseteq A, A / I$ is bounded in $R / I$ whenever $A / I \neq 0$;

(3) for any left annihilator $B$ and any ideal $J$ in $R$ with $J \subseteq B, B / J$ is bounded in $R / J$ whenever $B / J \neq 0$.

Proof. (1) $\Rightarrow(2)$ and $(1) \Rightarrow(3)$ are obvious.

$(2) \Rightarrow(1)$ : Assume on the contrary that there is a right annihilator $A$ with $R A \nsubseteq A$. We apply the proving method of [7, Proposition 6]. Let $H$ be the sum of all ideals contained in $A$. Then $A / H \neq 0$, and then $A / H$ is bounded; hence there is a nonzero ideal $K / H$ of $R / H$ contained in $A / H$, a contradiction. The proof of (3) $\Rightarrow(1)$ is similar.

Based on this remark we introduce the following concepts which generalise strongly one-sided bounded rings and IFP rings. A ring $R$ is called right (resp. left) $A B$ if every essential right (resp. left) annihilator of $R$ is bounded; i.e. it contains a non-zero ideal of $R$. A ring $R$ is called strongly right (resp. left) $A B$ if every non-zero right (resp. left) annihilator of $R$ is bounded; $R$ is called (strongly) $A B$ if $R$ is (strongly) right and (strongly) left AB. Obviously strongly right bounded rings and IFP rings are both strongly right $\mathrm{AB}$, but the converses need not be true in either case as we see in the next section. We in this paper concentrate on strongly one-sided $\mathrm{AB}$ rings.

We study the properties of strongly one-sided $\mathrm{AB}$ rings and the relationship between strongly one-sided AB rings and related rings. As an application of strongly one-sided AB rings, we observe the von Neumann regularity of SF rings. A ring $R$ is called von Neumann regular (simply regular) if for any $a \in R, a=a b a$ for some $b \in R$. A ring $R$ is called strongly regular if for any $a \in R, a=a^{2} b$ for some $b \in R$. A ring is strongly regular if and only if it is abelian regular if and only if it is reduced regular if and only if it is (one-sided) duo regular [15, Theorems 3.2 and 3.5]. A ring $R$ is called a right (resp. left) $S F$ if every simple right (resp. left) $R$-module is flat. A ring $R$ is regular if and only if all right (left) $R$-modules are flat [15, Corollary 1.3]. 
Ramamurthi [29] initiated the study of the von Neumann regularity of SF rings, asking whether a right and left SF ring is necessarily von Neumann regular. This question has drawn the attention of many authors [e.g. 9, 16, 30, 33, 37, 38]. For example, Zhang and Du [38] proved that a ring is strongly regular if and only if it is strongly right bounded and right SF. Also Ramamurthi [29] proved that a ring is strongly regular if and only if it is IFP and right SF. Some affirmative situations have been found, but the question remains open.

We lastly extend the class of strongly one-sided AB rings, concerning some conditions and some ring extensions.

2. Basic forms of strongly one-sided $\mathbf{A B}$ rings. In this section we study basic examples of strongly one-sided $\mathrm{AB}$ rings. We first construct a typical example of strongly one-sided $\mathrm{AB}$ rings. Use $E_{i j}$ for the matrix with $(i, j)$-entry 1 and elsewhere 0 . Given any ring $R$ we consider a ring extension

$$
R_{n}=\left\{\left(\begin{array}{cccccc}
a & a_{12} & a_{13} & \cdots & a_{1(n-1)} & a_{1 n} \\
0 & a & a_{23} & \cdots & a_{2(n-1)} & a_{2 n} \\
0 & 0 & a & \cdots & a_{3(n-1)} & a_{3 n} \\
\vdots & \vdots & \vdots & \ddots & \vdots & \vdots \\
0 & 0 & 0 & \cdots & a & a_{(n-1) n} \\
0 & 0 & 0 & \cdots & 0 & a
\end{array}\right) \mid a, a_{i j} \in R\right\},
$$

where $n(\geq 2)$ is a positive integer.

LEMMA 2.1. Let $R$ be $a$ ring and $0 \neq a \in R$. Then $a$ is right (resp. left) regular if and only if

$$
\left(\begin{array}{cccc}
a & a_{12} & \cdots & a_{1 n} \\
0 & a & \cdots & a_{2 n} \\
\vdots & \vdots & \ddots & \vdots \\
0 & 0 & \cdots & a
\end{array}\right) \in R_{n}
$$

is right (resp. left) regular.

Proof. Suppose that $a \in R$ is right regular, and let

$$
A=\left(\begin{array}{cccc}
a & a_{12} & \cdots & a_{1 n} \\
0 & a & \cdots & a_{2 n} \\
\vdots & \vdots & \ddots & \vdots \\
0 & 0 & \cdots & a
\end{array}\right) \in R_{n}
$$

We proceed by induction on $n$. Put $\left(\begin{array}{cc}a & a_{12} \\ 0 & a\end{array}\right)\left(\begin{array}{cc}b & b_{12} \\ 0 & b\end{array}\right)=0$ for some $\left(\begin{array}{cc}b & b_{12} \\ 0 & b\end{array}\right) \in R_{2}$. Then $a b=0$ and $a b_{12}+a_{12} b=0$. Since $a$ is right regular, $b=0$, and so $b_{12}=0$; hence $\left(\begin{array}{cc}a & a_{12} \\ 0 & a\end{array}\right)$ is right regular. Next let

$$
A B=0 \text { for } B=\left(\begin{array}{cccc}
b & b_{12} & \cdots & b_{1 n} \\
0 & b & \cdots & b_{2 n} \\
\vdots & \vdots & \ddots & \vdots \\
0 & 0 & \cdots & b
\end{array}\right) \in R_{n}
$$


Then we get

$$
\left(\begin{array}{cccc}
a & a_{12} & \cdots & a_{1(n-1)} \\
0 & a & \cdots & a_{2(n-1)} \\
\vdots & \vdots & \ddots & \vdots \\
0 & 0 & \cdots & a
\end{array}\right)\left(\begin{array}{cccc}
b & b_{12} & \cdots & b_{1(n-1)} \\
0 & b & \cdots & b_{2(n-1)} \\
\vdots & \vdots & \ddots & \vdots \\
0 & 0 & \cdots & b
\end{array}\right)=0
$$

By the induction hypothesis we obtain $b=0$ and $b_{i j}=0$ for $1 \leq i, j \leq n-$ 1 ; hence $a b_{1 n}+a_{12} b_{2 n}+\cdots+a_{1(n-1)} b_{(n-1) n}=0, a b_{2 n}+a_{23} b_{3 n}+\cdots+a_{2(n-2)} b_{(n-2) n}+$ $a_{2(n-1)} b_{(n-1) n}=0, \ldots, a b_{(n-3) n}+a_{(n-3)(n-2)} b_{(n-2) n}+a_{(n-3)(n-1)} b_{(n-1) n}=0, a b_{(n-2) n}+$ $a_{(n-2)(n-1)} b_{(n-1) n}=0, a b_{(n-1) n}=0$. From $a b_{(n-1) n}=0$ we get $b_{(n-1) n}=0$, and then from $a b_{(n-2) n}+a_{(n-2)(n-1)} b_{(n-1) n}=0$ we also get $b_{(n-2) n}=0$. Inductively we obtain $b_{i n}=0$ for $i=1,2, \ldots, n-1$, concluding that $A$ is right regular. Conversely assume that $A$ is right regular, and let $a b=0$ for some $b \in R$. Then from $A\left(b E_{1 n}\right)=0$ we have $b=0$. Thus $a$ is regular. The proof of left case is similar.

With the help of Lemma 2.1 we construct a typical example of strongly one-sided $\mathrm{AB}$ rings in the following.

THEOREM 2.2. A ring $R$ is strongly right (resp. left) $A B$ if and only if $R_{n}$ is strongly right (resp. left) $A B$ for any $n \geq 2$.

Proof. Suppose that $R$ is strongly right $\mathrm{AB}$ and $X \subseteq R_{n}$ with $r_{R_{n}}(X) \neq 0$. Then any diagonal in matrices in $X$ is not right regular by Lemma 2.1. Let $Y$ be the set of all elements in $R$, which occur as diagonal entries of elements in $X$. If $Y=0$, then $r_{R_{n}}(X)$ contains a non-zero ideal $R E_{1 n}$ of $R_{n}$. Next we suppose $Y \neq 0$ and let $a$ be any in $Y$. Take

$$
0 \neq\left(\begin{array}{cccc}
b & b_{12} & \cdots & b_{1 n} \\
0 & b & \cdots & b_{2 n} \\
\vdots & \vdots & \ddots & \vdots \\
0 & 0 & \cdots & b
\end{array}\right) \text { in } r_{R_{n}}(X)
$$

We will show $r_{R}(Y) \neq 0$.

(Case 1) If $b \neq 0$, then $r_{R}(Y) \neq 0$;

(Case 2) Assume $b=0$, and proceed by induction on $n$.

Set $n=2$. Then clearly $0 \neq b_{12} \in r_{R}(Y)$.

Set $n=3$. If $b_{23} \neq 0$, then we are done. If $b_{23}=0$, then we get $a b_{13}=0$. Here if $b_{13} \neq 0$, then we are done; otherwise we get $0 \neq b_{12} \in r_{R}(Y)$. Consequently $r_{R}(Y) \neq 0$. Set

$$
\left(\begin{array}{cccc}
a & a_{12} & \cdots & a_{1 n} \\
0 & a & \cdots & a_{2 n} \\
\vdots & \vdots & \ddots & \vdots \\
0 & 0 & \cdots & a
\end{array}\right)\left(\begin{array}{cccc}
b & b_{12} & \cdots & b_{1 n} \\
0 & b & \cdots & b_{2 n} \\
\vdots & \vdots & \ddots & \vdots \\
0 & 0 & \cdots & b
\end{array}\right)=0 \text { for }\left(\begin{array}{cccc}
a & a_{12} & \cdots & a_{1 n} \\
0 & a & \cdots & a_{2 n} \\
\vdots & \vdots & \ddots & \vdots \\
0 & 0 & \cdots & a
\end{array}\right) \in X
$$

If $b_{(n-1) n} \neq 0$, then we are done; otherwise we have $a b_{(n-2) n}=0$. Check whether $b_{(n-2) n}=0$. Inductively we can check whether there is a non-zero $b_{\text {in }} \in r_{R}(Y)$ for some $i \in\{1,2, \ldots, n-1\}$; if every $b_{i n}$ is zero, then there is a non-zero $b_{i j} \in r_{R}(Y)$ 
for $i \in\{1,2, \ldots, n-2\}$ and $j \in\{1,2, \ldots, n-1\}$, by the induction hypothesis. Thus $r_{R}(Y) \neq 0$.

Now since $R$ is strongly right $\mathrm{AB}$, there is a non-zero ideal $I$ of $R$ with $I \subseteq r_{R}(Y)$. Then $r_{R_{n}}(X)$ contains a non-zero ideal $I E_{1 n}$ of $R_{n}$. Thus $R_{n}$ is strongly right AB.

Conversely suppose that $R_{n}$ is strongly right $\mathrm{AB}$ and $V \subseteq R$ with $r_{R}(V) \neq 0$. Let $W=\left\{a I_{n} \mid a \in V\right\} \subseteq R_{n}$, where $I_{n}$ is the $n \times n$ identity matrix. Then $r_{R_{n}}(W) \neq$ 0 because $W U=0$ for any non-zero matrix $U$ in $R_{n}$ with entries in $r_{R}(V)$. Since $R_{n}$ is strongly right $\mathrm{AB}$, there exists a non-zero ideal $J$ of $R_{n}$ such that $r_{R_{n}}(W) \supseteq$ $J$. Set $K=\{c \in R \mid c$ is an entry of a matrix in $J\}$. Then $K$ is a non-zero ideal of $R$ from the computations $\left(a I_{n}\right)\left(r I_{n}\right) C=0$ and $\left(a I_{n}\right) C\left(r I_{n}\right)=0$ for $a \in V, r \in R$ and $C \in$ $J$. Moreover $a K=0$ for all $a \in V$ from $\left(a I_{n}\right) J=0$, entailing $r_{R}(X) \supseteq K$. Thus $R$ is strongly right $\mathrm{AB}$. The proof of the left case is similar.

REMARK. Let $U$ and $L$ be the $n \times n$ upper and lower triangular matrix rings over a ring, respectively; $U$ and $L$ are isomorphic, via the correspondence $\left(a_{i j}\right) \mapsto$ $\left(b_{s t}\right)$ with $b_{s t}=a_{(n-s+1)(n-t+1)} ; R_{n}$ and $R_{n}^{\prime}$ are also isomorphic under the preceding correspondence, where $R_{n}^{\prime}$ is the image of $R_{n}$. Thus every property obtained for $U$ (resp. $R_{n}$ ) is also true for $L$ (resp. $R_{n}^{\prime}$ ).

We use $G F\left(p^{n}\right)$ to denote the Galois field of order $p^{n}$. Given a ring $R$ we denote the order of $R$ and the characteristic of $R$ by $|R|$ and $C h(R)$, respectively. Strongly right bounded rings and IFP rings are both strongly right $\mathrm{AB}$, but the converse need not be true as we see in the following.

EXAMPLE 2.3. (1) Let $R$ be any ring and $M$ be the $2 \times 2$ matrix ring over $R$. Each of $r_{M}\left(E_{11}\right)=R E_{21}=R E_{22}$ and $\ell_{M}\left(E_{11}\right)=R E_{12}+R E_{22}$ cannot contain a non-zero ideal of $M$. So $M$ is neither strongly right nor strongly left AB. Let $U$ be the $2 \times 2$ upper triangular matrix ring over $R$. Each of $r_{U}\left(E_{11}\right)=R E_{22}$ and $\ell_{U}\left(E_{22}\right)=R E_{11}$ cannot contain a non-zero ideal of $U$. So $U$ is neither strongly right nor strongly left AB. By similar computations, any $n \times n$ (upper or lower triangular) matrix ring is neither strongly right nor strongly left $\mathrm{AB}$.

(2) There exists a strongly right $\mathrm{AB}$ ring that is not strongly right bounded. Let $R$ be a strongly right $\mathrm{AB}$ ring and consider $R_{3}$ over $R ; R_{3}$ is strongly right $\mathrm{AB}$ by Theorem 2.2, but it is not strongly right bounded by the following: $E_{23} R_{3}=R E_{23}$ and $R_{3} E_{12}=R E_{12}$ cannot contain any non-zero ideal of $R_{3}$. By this computation, $R_{n}$ ( $n \geq 3$ ), over any ring $R$, is neither strongly right nor strongly left bounded.

(3) There exists a strongly right AB ring that is not IFP. Let $R$ be a strongly right $\mathrm{AB}$ ring and consider $R_{4}$ over $R ; R_{4}$ is strongly right $\mathrm{AB}$ by Theorem 2.2 , but it is not IFP by [23, Example 1.3].

(4) There are no containing relations between the classes of strongly right bounded rings and IFP rings. Let $R$ be a reduced ring, and consider $R_{3}$ over $R ; R_{3}$ is IFP by [23, Proposition 1.2], but it is not strongly right bounded by the computation in (2).

Next let $R=\left(\begin{array}{cc}G F(2) & G F(2) \\ 0 & \mathbb{Z}\end{array}\right)$, where $\mathbb{Z}$ is the ring of integers. Note that each non-zero right ideal of $R$ is one of the following: $\left(\begin{array}{cc}G F(2) & G F(2) \\ 0 & n \mathbb{Z}\end{array}\right),\left(\begin{array}{cc}G F(2) & G F(2) \\ 0 & 0\end{array}\right),\left(\begin{array}{cc}0 & G F(2) \\ 0 & n \mathbb{Z}\end{array}\right),\left(\begin{array}{cc}0 & G F(2) \\ 0 & 0\end{array}\right)$ and $\left(\begin{array}{cc}0 & 0 \\ 0 & n \mathbb{Z}\end{array}\right)$ for $n=1,2, \ldots$. Since $n \mathbb{Z} E_{22}$ contains an ideal $2 n \mathbb{Z} E_{22}$ of $R$ and remainders are two-sided, $R$ is strongly right bounded. However $\mathbb{Z} E_{22}=r_{R}\left(E_{11}\right)$ is not two-sided, and thus $R$ is not IFP.

(5) There is a domain that is neither strongly right nor strongly left bounded ring. Let $R$ be the first Weyl algebra over a field $F$ of characteristic zero. Then $R$ is a simple right Noetherian domain which is not a division ring. Thus $R$ is reduced, and so $R$ 
is strongly $\mathrm{AB}$. There is $0 \neq a \in R$ such that $a R \neq R$, since $R$ is not a division ring. Assume that there exists a non-zero ideal $I$ of $R$ such that $I \subseteq b R$. Then we have $R=I \subseteq b R$, a contradiction; hence $R$ is not strongly right bounded. Similarly $R$ is also not strongly left bounded.

We next observe the basic structure of strongly one-sided $\mathrm{AB}$ rings in the following several results.

The following results are obtained from Eldridge [12].

Lemma 2.4. (1) [12, Proposition] Let $R$ be a finite non-commutative ring. If the order of $R$ is $p^{3}$, with $p$ being a prime, then $R$ is isomorphic to the $2 \times 2$ upper triangular matrix ring over $G F(p)$.

(2) [12, Theorem] Let $R$ be a finite ring of order $m$. If $m$ has a cube-free factorisation, then $R$ is a commutative ring.

EXAMPLE 2.5. (1) By Lemma 2.4(1,2) every minimal non-commutative ring is isomorphic to the $2 \times 2$ upper triangular matrix ring over $G F(2)$. So by Theorem 2.2 and Example 2.3(1,2), $R_{3}$ over $G F(2)$ is a minimal non-commutative strongly $\mathrm{AB}$ ring that is neither strongly right nor strongly left bounded.

(2) There is a strongly right bounded ring but not right duo. We refer to the ring in [34, Remark 4]. Let $S$ and $T$ be the $2 \times 2$ upper and lower triangular matrix rings over $G F(p)$ respectively. Set $R$ be the subring of $S \oplus T$ consisting of all the elements of the form $\left(\left(\begin{array}{cc}a & c \\ 0 & b\end{array}\right),\left(\begin{array}{ll}a & 0 \\ d & b\end{array}\right)\right)$. Then $R$ is strongly right bounded but not right duo as can be seen by the right ideal of $R$ generated by $\left(E_{11}, E_{11}\right)$. Note that $R$ is a minimal noncommutative strongly right (hence left by [34, Proposition 2]) bounded ring of order 16 when $p=2$, with the help of Example 2.3(1) and Lemma 2.4.

(3) We refer to the three rings in [35, Example 2] as follows: Let $S\{x, y\}$ be the free algebra generated by $x, y$ over a ring $S$.

Set $\quad B_{1}=G F(2)\{x, y\} /\left(x^{3}, y^{3}, y x, x^{2}-x y, y^{2}-x y\right), \quad$ where $\quad\left(x^{3}, y^{3}, y x, x^{2}-\right.$ $\left.x y, y^{2}-x y\right)$ is the ideal of $G F(2)\{x, y\}$ generated by $x^{3}, y^{3}, y x, x^{2}-x y, y^{2}-x y$.

Let $\mathbb{Z}_{4}=\{\overline{0}, \overline{1}, \overline{2}, \overline{3}\}$ be the ring of integers modulo 4 and

$$
B_{2}=\mathbb{Z}_{4}\{x, y\} /\left(x^{3}, y^{3}, y x, x^{2}-x y, x^{2}-\overline{2}, y^{2}-\overline{2}, \overline{2} x, \overline{2} y\right),
$$

where $\left(x^{3}, y^{3}, y x, x^{2}-x y, x^{2}-\overline{2}, y^{2}-\overline{2}, \overline{2} x, \overline{2} y\right)$ is the ideal of $\mathbb{Z}_{4}\{x, y\}$ generated by $x^{3}, y^{3}, y x, x^{2}-x y, x^{2}-\overline{2}, y^{2}-\overline{2}, \overline{2} x, \overline{2} y$.

Let $B_{3}$ be the ring of all matrices of the form $\left(\begin{array}{cc}a & b \\ 0 & a^{2}\end{array}\right)$ over $G F\left(2^{2}\right)$.

Then each $B_{i}$ is a non-commutative duo ring of order 16 by [34, Proposition 3]. Note that $C h\left(B_{1}\right)=2=C h\left(B_{3}\right), C h\left(B_{2}\right)=4$ and $\left|J\left(B_{1}\right)\right|=8,\left|J\left(B_{3}\right)\right|=4$. Thus $B_{1} \nsucceq$ $B_{2}, B_{1} \nsucceq B_{3}$ and $B_{2} \nsucceq B_{3}$. Any minimal non-commutative duo ring is isomorphic to $B_{i}$ for some $i=1,2,3$ by [35, Theorem 3].

(4) Consider two subrings of the $4 \times 4$ matrix ring over $G F\left(p^{n}\right)$. Define

$$
C_{1}=\left\{\left(\begin{array}{cccc}
a & 0 & 0 & c \\
0 & a & 0 & 0 \\
0 & 0 & b & d \\
0 & 0 & 0 & b
\end{array}\right) \mid a, b, c, d \in G F\left(p^{n}\right)\right\}
$$


and

$$
\begin{gathered}
C_{2}=\left\{\left(\begin{array}{llll}
a & 0 & 0 & 0 \\
0 & a & 0 & 0 \\
0 & 0 & b & 0 \\
c & 0 & d & b
\end{array}\right) \mid a, b, c, d \in G F\left(p^{n}\right)\right\} \\
\left(\begin{array}{cccc}
0 & 0 & 0 & G F\left(p^{n}\right) \\
0 & 0 & 0 & 0 \\
0 & 0 & 0 & 0 \\
0 & 0 & 0 & 0
\end{array}\right) \text { and }\left(\begin{array}{cccc}
0 & 0 & 0 & 0 \\
0 & 0 & 0 & 0 \\
0 & 0 & 0 & G F\left(p^{n}\right) \\
0 & 0 & 0 & 0
\end{array}\right)
\end{gathered}
$$

are non-zero ideals of $C_{1}$; hence every non-zero right annihilator contains at least one of them, and so $C_{1}$ is strongly right $\mathrm{AB}$. However

$$
\ell_{C_{1}}\left(\left(\begin{array}{cccc}
0 & 0 & 0 & 0 \\
0 & 0 & 0 & 0 \\
0 & 0 & 1 & 0 \\
0 & 0 & 0 & 1
\end{array}\right)\right)=\left\{\left(\begin{array}{cccc}
a & 0 & 0 & 0 \\
0 & a & 0 & 0 \\
0 & 0 & 0 & 0 \\
0 & 0 & 0 & 0
\end{array}\right) \mid a \in G F\left(p^{n}\right)\right\}
$$

is a minimal left ideal of $C_{1}$ that is not an ideal, and so it is not strongly left $\mathrm{AB}$. Moreover $C_{1}$ is not strongly right bounded, since

$$
\left(\begin{array}{llll}
0 & 0 & 0 & 1 \\
0 & 0 & 0 & 0 \\
0 & 0 & 0 & 1 \\
0 & 0 & 0 & 0
\end{array}\right) C_{1}
$$

is a minimal right ideal of $C_{1}$ that is not an ideal.

By a similar computation we get that $C_{2}$ is strongly left $\mathrm{AB}$ but neither strongly right $\mathrm{AB}$ nor strongly left bounded.

In the next theorem we see all minimal non-commutative strongly right $\mathrm{AB}$ rings up to isomorphism, with the help of [35, Theorem 3].

THEOREM 2.6. (1) If $R$ is a minimal non-commutative strongly right $A B$ ring, then $R$ is isomorphic to $A_{k}$ for some $k \in\{1,2,3,4,5,6\}$, where $A_{i}$ is the ring $B_{i}$ in Example 2.5(3) for $i=1,2,3 ; A_{4}$ is $R_{3}$ over $G F(2) ; A_{5}$ is the ring $R$ in Example 2.5(2); $A_{6}=C_{1}$ with $p^{n}=2$ in Example 2.5(4).

(2) If $R$ is a minimal non-commutative strongly left $A B$ ring, then $R$ is isomorphic to $A_{k}$ for some $k \in\{1,2,3,4,5,6\}$, where $A_{i}$ is the ring $B_{i}$ in Example 2.5(3) for $i=1,2,3$; $A_{4}$ is $R_{3}$ over $G F(2) ; A_{5}$ is the ring $R$ in Example 2.5(2); $A_{6}=C_{2}$ with $p^{n}=2$ in Example $2.5(4)$.

Proof. (1) Any minimal non-commutative strongly right AB ring must be of order 16 by Theorem 2.2 (consider $R_{3}$ over $G F(2)$ ), Example 2.3(1) and Lemma 2.4(1, 2). Let $R$ be a minimal non-commutative strongly right $\mathrm{AB}$ ring.

(The case of local rings) We have three cases of $|J(R)|=2,|J(R)|=4$ or $|J(R)|=8$. Then $R / J(R)$ is clearly a field, and by the argument in [35, Theorem 3], $J(R)$ is a vector space over $R / J(R)$. If $|J(R)|=2$, then $\frac{R}{J(R)} \cong G F\left(2^{3}\right)$, and so $|J(R)| \geq 8$, a contradiction. So have two cases of $|J(R)|=4$ and $|J(R)|=8$. 
Suppose $|J(R)|=8$. Then $\frac{R}{J(R)} \cong G F(2)$, and by [25, Theorem 2.3.6], $J(R)$ has a basis $\{a, b, c\}$ over $G F(2)$ such that $a^{2}=a b=c, b a=0, b^{2}=0$, or $a^{2}=a b=c, b a=0$, $b^{2}=c$.

Assume that $a^{2}=a b=c, b a=0, b^{2}=c$. Then [35, Theorem 3] implies that $R \cong$ $A_{1}$ if $C h(R)=2$ and $R \cong A_{2}$ if $C h(R)=4$.

Assume that $a^{2}=a b=c, b a=0, b^{2}=0$. Then $b R$ is a minimal right ideal but not an ideal, and so $R$ is not strongly right bounded. Consider $A_{4}$. Then

$$
J\left(A_{4}\right)=\left(\begin{array}{ccc}
0 & G F(2) & G F(2) \\
0 & 0 & G F(2) \\
0 & 0 & 0
\end{array}\right)
$$

and $R \cong A_{4}$, letting

$$
a=\left(\begin{array}{lll}
0 & 1 & 0 \\
0 & 0 & 1 \\
0 & 0 & 0
\end{array}\right), b=\left(\begin{array}{lll}
0 & 0 & 0 \\
0 & 0 & 1 \\
0 & 0 & 0
\end{array}\right) \text { and } c=\left(\begin{array}{lll}
0 & 0 & 1 \\
0 & 0 & 0 \\
0 & 0 & 0
\end{array}\right)
$$

Suppose $|J(R)|=4$. Then $\frac{R}{J(R)} \cong G F\left(2^{2}\right)$, and by [35, Theorem 3] $R \cong A_{3}$.

(The case of non-local rings) We apply in part the proof of [34, Proposition 2]. First assume $R=A \oplus B$; then $|A|=2^{3}$ or $|B|=2^{3}$ by Lemma 2.4(2), since $R$ is noncommutative. But by Lemma 2.4(1) and Example 2.3(1), any case is impossible; hence $R$ is indecomposable. Since $R$ is finite and non-local, $R$ contains a non-trivial idempotent $e$ (i.e. $e \neq 0$ and $e \neq 1$ ). Put $f=1-e$ and consider the Pierce decomposition $R=$ $\left(\begin{array}{ccc}e R e & e R f \\ f & R e & f R f\end{array}\right)$.

Suppose that $e R f \neq 0$ and $f R e \neq 0$. Then $|e R e|=|e R f|=|f R e|=|f R f|=2$. If $e R f R e \neq 0$, then $e R f R e=e R e$ and $r_{R}\left(\left(\begin{array}{cc}0 & e R f \\ 0 & 0\end{array}\right)\right)=\left(\begin{array}{cc}e R e & e R f \\ 0 & 0\end{array}\right)$ cannot contain a non-zero ideal of $R$, a contradiction to the strong right ABness of $R$. If $f \operatorname{Re} R f \neq 0$, then we also have a contradiction by a similar method. Consequently $e R f R e=0=f R e R f$. Then $\left(\begin{array}{cc}0 & e R f \\ 0 & 0\end{array}\right)$ and $\left(\begin{array}{cc}0 & 0 \\ f R e & 0\end{array}\right)$ are ideals of $R$; hence $R$ is strongly right bounded. In this case $R \cong A_{5}$ when $p=2$ with $e=\left(\left(\begin{array}{ll}1 & 0 \\ 0 & 0\end{array}\right),\left(\begin{array}{ll}1 & 0 \\ 0 & 0\end{array}\right)\right)$ and $f=\left(\left(\begin{array}{ll}0 & 0 \\ 0 & 1\end{array}\right),\left(\begin{array}{ll}0 & 0 \\ 0 & 1\end{array}\right)\right)$.

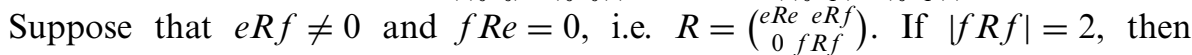
$r_{R}\left(\left(\begin{array}{ll}e & 0 \\ 0 & 0\end{array}\right)\right)=\left(\begin{array}{cc}0 & 0 \\ 0 & f R f\end{array}\right)$, but this is a minimal right ideal of $R$ that does not contain a non-zero ideal of $R$, a contradiction to the strong right ABness of $R$. So we must assume $|f R f|=2^{2}$. If $f R f$ is decomposable, say $f R f=D_{1} \oplus D_{2}$, then $e R f D_{i} \neq 0$ for some $i=1,2$ because $e R f \neq 0$, say $e R f D_{1} \neq 0$. Hence $R$ has a minimal right ideal $r_{R}\left(\left(\begin{array}{cc}e & 0 \\ 0 & D_{2}\end{array}\right)\right)=\left(\begin{array}{ll}0 & 0 \\ 0 & D_{1}\end{array}\right)$ that is not an ideal, and thus $R$ is not strongly right AB. Consequently $f R f$ is a local ring. But $|J(f R f)|=2$. For, if $J(f R f)=0$, then $f R f$ is a field and $|e R f| \geq 4$, since $e R f$ is a vector space over $f R f$, a contradiction. Note $J(f R f)^{2}=0$. For if $J(f R f)^{2} \neq 0$, then $J(f R f)^{2}=J(f R f)$, and so $J(f R f)$ is nonnilpotent, a contradiction. Since $\left(\begin{array}{ll}0 & x \\ 0 & y\end{array}\right) R$ is a minimal right ideal of $R$ but not ideal, $R$ is not strongly right bounded, where $0 \neq x \in e R f$ and $0 \neq y \in J(f R f)$. In this case $R \cong A_{6}$ with

$$
e=\left(\begin{array}{llll}
1 & 0 & 0 & 0 \\
0 & 1 & 0 & 0 \\
0 & 0 & 0 & 0 \\
0 & 0 & 0 & 0
\end{array}\right) \text { and } f=\left(\begin{array}{llll}
0 & 0 & 0 & 0 \\
0 & 0 & 0 & 0 \\
0 & 0 & 1 & 0 \\
0 & 0 & 0 & 1
\end{array}\right)
$$


(2) The proof is similar to that of (1).

REMARK. The proof of the case of non-local rings in Theorem 2.6 is applicable for any prime $p$ in place of 2 .

COROLlARY 2.7. If $R$ is a minimal non-commutative strongly right bounded ring, then $R$ is isomorphic to $A_{i}$ for some $i \in\{1,2,3,5\}$, where $A_{i}$ denotes the rings in Theorem 2.6.

Proof. In the proof of Theorem 2.6, $A_{i}$ is right duo for $i \in\{1,2,3\} ; A_{5}$ is strongly right bounded; and the rings $A_{4}, A_{6}$ are not strongly right bounded.

3. Properties of strongly one-sided AB rings and related rings. In this sections we study the properties of strongly one-sided $\mathrm{AB}$ rings and the relationship between strongly one-sided $\mathrm{AB}$ rings and related rings. As an application of strongly one-sided $\mathrm{AB}$ rings, we observe the von Neumann regularity of right $\mathrm{SF}$ rings under some specific conditions (e.g. strong right ABness).

LeMma 3.1. Let $R_{i}$ be a ring for $i \in I$ and $D$ be the direct product of $R_{i}$ 's.

(1) Every $R_{i}$ is strongly right (resp. left) $A B$ if and only if $D$ is strongly right (resp. left) $A B$. This result also holds for direct sums (possibly without identity).

(2) Every $R_{i}$ is strongly right (resp. left) bounded if and only if D is strongly right (resp. left) bounded. This result also holds for direct sums (possibly without identity).

Proof. (1) Suppose that $R_{i}$ is strongly right $\mathrm{AB}$ for each $i \in I$. Let $X \subseteq D$ with $r_{D}(X) \neq 0$ and $Y_{i}$ be the set of all the $i$ th compontents of the sequences in $X$. From $r_{D}(X) \neq 0$ there is $j \in I$ such that $r_{R_{j}}\left(Y_{j}\right) \neq 0$. Since $R_{j}$ is strongly right AB there is a non-zero ideal $K$ of $R_{j}$ with $K \subseteq r_{R_{j}}\left(Y_{j}\right)$. Set $H$ be the subset of $D$ such that $e_{j} H=K$ and $e_{k} H=0$ for $k \neq j$, where $e_{i}$ is the sequence in $D$ with $i$ th component $1_{R_{i}}$ and elsewhere zero. Since $H$ is a non-zero ideal of $D$ with $X H=0, D$ is strongly right AB. Conversely suppose that $D$ is strongly right $\mathrm{AB}$ and $R_{j}$ is not strongly right $\mathrm{AB}$ for some $j \in I$. Take $X \subseteq R_{j}$ such that $r_{R_{j}}(X)$ is non-zero, and this does not contain any non-zero ideal of $R_{j}$. Consider $Y \subseteq D$ such that $e_{j} Y=X$ and $e_{k} Y=\left\{1_{R_{k}}\right\}$ for $k \neq j$. Then $e_{j} r_{D}(Y)=r_{R_{j}}(X)$ and $e_{k} r_{D}(Y)=0$ for $k \neq j$; hence $r_{D}(Y)$ does not contain any non-zero ideal of $D$, a contradiction. The direct sum case and the left case can be proved similarly. The proof of (2) is similar to (1).

From Lemma 3.1 it is natural to ask whether the class of strongly one-sided AB rings is closed under subrings and factor rings. However the answer is negative by the following.

ExAMPLE 3.2. Let $R$ be the strongly right $\mathrm{AB}$ ring $C_{1}$ in Example 2.5(4), and consider the subring

$$
S=\left\{\left(\begin{array}{cccc}
a & 0 & 0 & c \\
0 & a & 0 & 0 \\
0 & 0 & b & 0 \\
0 & 0 & 0 & b
\end{array}\right) \mid a, b, c \in G F\left(p^{n}\right)\right\}
$$


of $R$. Then $S$ is not strongly right $\mathrm{AB}$ because

$$
r_{S}\left(\left(\begin{array}{llll}
1 & 0 & 0 & 0 \\
0 & 1 & 0 & 0 \\
0 & 0 & 0 & 0 \\
0 & 0 & 0 & 0
\end{array}\right)\right)=\left\{\left(\begin{array}{cccc}
0 & 0 & 0 & 0 \\
0 & 0 & 0 & 0 \\
0 & 0 & b & 0 \\
0 & 0 & 0 & b
\end{array}\right) \mid b \in G F\left(p^{n}\right)\right\}
$$

is a minimal right ideal of $S$ that is not an ideal. Next consider the factor ring $R / I$ by the ideal

$$
I=\left\{\left(\begin{array}{cccc}
0 & 0 & 0 & 0 \\
0 & 0 & 0 & 0 \\
0 & 0 & 0 & d \\
0 & 0 & 0 & 0
\end{array}\right) \mid d \in G F\left(p^{n}\right)\right\}
$$

$R / I$ is isomorphic to the preceding $\operatorname{ring} S$ and so is not strongly right $\mathrm{AB}$. The left cases can be shown similarly.

The IFPness is left-right symmetric, but the strong ABness is not left-right symmetric by Example 2.5(4) in spite of being finite rings. The strong boundedness and duoness are also not left-right symmetric by the following.

EXAMPLE 3.3 (1) Let $R$ be the ring in Example 2.3(4). Then $R$ is strongly right bounded. But $\ell_{R}\left(\left(\begin{array}{ll}0 & 0 \\ 0 & 1\end{array}\right)\right)=\left(\begin{array}{cc}G F(2) & 0 \\ 0 & 0\end{array}\right)$ cannot contain a right ideal of $R$, concluding that $R$ is not strongly left AB (hence not strongly left bounded).

(2) Let $F$ be a field and $F(x)$ be the field of rational functions over $F$. Due to Chatters and Xue [8], set $R=F(x) \oplus F(x)$ to be an additive group with the multiplication $\left(f_{1}(x), g_{1}(x)\right)\left(f_{2}(x), g_{2}(x)\right)=\left(f_{1}(x) f_{2}(x), f_{1}\left(x^{2}\right) g_{2}(x)+g_{1}(x) f_{2}(x)\right)$. Then $R$ is right duo but not left duo by the computation in the example in [8].

In the following lemma we check some conditions, obtained by Chatters, Courter and Xue, under which the strong boundedness and duoness can be left-right symmetric. We denote by $c(M)$ the composition length of a module $M$ over a ring $R$.

Lemma 3.4 (1) [34, Proposition 3] A finite right duo ring is left duo.

(2) [10, Theorem 2.2] A right Artinian right duo ring $R$ is left duo if $c\left(R_{R}\right)=c\left({ }_{R} R\right)$.

(3) [10, Corollary 2.3] A right duo ring is left duo when it is a finite-dimensional algebra over a field.

(4) [34, Theorem] If $R$ is a strongly right bounded finite ring and $|R|$ has no factors of the form $p^{5}$, then $R$ is strongly left bounded.

(5) [34, Proposition 2] Let $R$ be a strongly right bounded ring with $|R|=p^{4}, p a$ prime. If $R$ is non-local, then $R$ is strongly left bounded.

(6) If $R$ is a local ring with nilpotent $J(R)$, then $R$ is strongly $A B$.

Proof. (6) Let $R$ be a local ring such that $J(R)^{k}=0$ and $J(R)^{k-1} \neq 0$. Let $X \subset R$ with $r_{R}(X) \neq 0$. Then $X \subseteq J(R)$, and so $X J(R)^{k-1}=0$; hence $R$ is strongly right AB. Similarly $R$ is strongly left $\mathrm{AB}$.

Local rings (especially of order $2^{4}$ ) with nilpotent Jacobson radical need not be strongly one-sided bounded as can be seen by the ring $R_{3}$ over $G F(2)$ in Example 2.3(2). But in the following we see situations for which finite strongly one-sided AB rings can be two-sided. 
THEOREM 3.5 (1) In Theorem 2.6, every $A_{i}$ is left-right symmetric for $i \in$ $\{1,2,3,4,5\}$.

(2) Let $R$ be a finite non-local strongly right $A B$ ring such that $|R|$ has no factors of the form $p^{5}$. Then $R$ is strongly right bounded if and only if $R$ is strongly left $A B$.

Proof. (1) By Lemma 3.4(1) $A_{i}$ is duo for $i=1,2,3 ; A_{4}$ is strongly AB by Lemma 3.4(6); and $A_{5}$ is strongly bounded by Lemma 3.4(4) or Lemma 3.4(5).

(2) It is well known that $R$ is a finite direct product of rings each of which has prime power order. Through Lemmas 2.4 and 3.1, we can assume $R$ is of order $p^{4}$ with $p$ a prime. If $R$ is strongly right bounded, then it is strongly left bounded by Lemma 3.4(5). Conversely if $R$ is strongly left $\mathrm{AB}$, then $R$ is strongly $\mathrm{AB}$, and so by Theorem 2.6(2) $R$ is isomorphic to the ring $R$ in Example 2.5(2); hence $R$ is strongly (right) bounded.

We next find more conditions under which the strong ABness or strong boundedness can be left-right symmetric.

Proposition 3.6. For a ring $R$ the following conditions are equivalent:

(1) $R$ is reduced;

(2) $R$ is semiprime and strongly right $A B$;

(3) $R$ is semiprime and strongly left $A B$;

(4) $R$ is semiprime and IFP.

Proof. (1) $\Rightarrow(4),(4) \Rightarrow(2)$ and $(4) \Rightarrow(3)$ are clear.

$(2) \Rightarrow(1)$ : Assume on the contrary that there exists $0 \neq a \in R$ satisfying $a^{2}=0$. Then $r_{R}\left(\ell_{R}(a)\right)$ is a non-zero right annihilator in $R$. Since $R$ is strongly right AB, there exists a non-zero ideal $I$ of $R$ such that $I \subseteq r_{R}\left(\ell_{R}(a)\right)$, i.e. $\ell_{R}(a) I=0$. We claim that $\ell_{R}(a) \cap I \neq 0$. If $I a=0$, then we are done. If $I a \neq 0$, then we also have $0 \neq I a \subseteq$ $\ell_{R}(a) \cap I$ because $(I a) a=0$. However $\left(\ell_{R}(a) \cap I\right)^{2} \subseteq \ell_{R}(a) I=0$, and so $\ell_{R}(a) \cap I=0$, since $R$ is semiprime, a contradiction. Thus $R$ is reduced. The proof of $(3) \Rightarrow(1)$ is similar.

A ring is called $P I$ if it satisfies a polynomial identity with coefficients in the ring of integers.

Proposition 3.7. For a PI ring $R$ the following conditions are equivalent:

(1) $R$ is reduced;

(2) $R$ is semiprime and strongly right $A B$;

(3) $R$ is semiprime and strongly left $A B$;

(4) $R$ is semiprime and IFP;

(5) $R$ is semiprime and strongly right bounded;

(5) $R$ is semiprime and strongly left bounded.

Proof. The equivalences of (1)-(4) come from Proposition 3.6; (1) $\Rightarrow(5)$ and (1) $\Rightarrow(6)$ are obtained by $[4$, Theorem $\mathrm{C}] ;(5) \Rightarrow(2)$ and $(6) \Rightarrow(3)$ are obvious.

The PI condition in Proposition 3.7 is not superfluous. The non-PI ring $R$ in Example 2.3(5) is a domain (hence reduced) which is neither strongly right nor strongly left bounded.

Due to Marks [27], a ring $R$ is called $N I$ if $N(R)=N^{*}(R)$, where $N^{*}(R)$ is the upper nilradical of $R$. It is obvious that a ring $R$ is NI if and only if $R / N^{*}(R)$ is reduced and that IFP rings are NI. A prime ideal $P$ of $R$ is called completely prime if $R / P$ is a domain. Hong and Kwak [18, Corollary 13] proved that a ring $R$ is NI if 
and only if every minimal strongly prime ideal of $R$ is completely prime. According to Köthe [24], a ring $R$ is called an I-ring if every non-nil right ideal of $R$ contains a nonzero idempotent. Algebraic algebras and $\pi$-regular rings are I-rings by [22, Proposition 9.4.1]. It is easy to check that Jacobson radicals of I-rings are nil.

LEMMA 3.8 (1) The definition of I-ring is left-right symmetric.

(2) A ring $R$ is an I-ring if and only if for each non-nilpotent element $x \in R$ there exists $0 \neq y \in R$ satisfying $y x y=y$.

(3) Let $R$ be an I-ring. If $R$ is strongly one-sided $A B$, then $N(R)=J(R)$.

(4) Strongly one-sided AB I-rings are NI.

Proof. (1) Let $R$ be an I-ring and $J$ be a non-nil left ideal of $R$. Let $a \in J$ be non-nilpotent. Then $a R$ is a non-nil right ideal, and so there is $0 \neq a b=a b a b$ for some $b \in R$, since $R$ is an I-ring. Consider $b a b a \in R a \subseteq J$; then $b a b a \neq 0$ and $(b a b a)(b a b a)=$ $b(a b a b a b) a=b a b a$.

(2) Let $R$ be an I-ring and $x=a, y=b a b$, where $a, b$ are the elements in (1). Then $y \neq 0$ and $y x y=(b a b) a(b a b)=b a b=y$. The converse is obvious.

(3) Let $R$ be an I-ring; then $J(R)$ is nil. Assume that there is $a \in N(R)$, say $a^{n}=0$ for some positive integer $n$, with $a \notin J(R)$. Then $a R$ is a non-nil right ideal of $R$. But $R$ is an I-ring; so $e \in a R$ for some non-zero $e^{2}=e \in R$. Since $R$ is strongly right AB, there exists a non-zero ideal $I$ of $R$ such that $I \subseteq r_{R}(1-e)=e R \subseteq a R$. Let $a r \in I$ and $e=a s$ for some $r, s \in R$. Then $a r=e a r$ and $s a r=e s a r$, since $I$ is two-sided. Thus $a r=$ ear $=$ asar $=$ aesar $=a^{2} s^{2} a r=a^{2} e s^{2} a r=\cdots=a^{n} s^{n} a r=0$, which is a contradiction. Thus $N(R) \subseteq J(R)$, entailing $N(R)=J(R)$. The proof of the left case is similar.

(4) If $R$ is strongly one-sided AB I-ring, then $R / N(R)$ is reduced by (3) with $N(R)=J(R)$.

Lemma 3.8(3, 4) is similar to [17, Proposition 3]. The converse of Lemma 3.8(3) need not be true as can be seen by the $2 \times 2$ upper triangular matrix ring over a division ring. The condition 'I-ring' in Lemma $3.8(3,4)$ is not superfluous by the following.

EXAMPLE 3.9. There is a strongly AB ring that is neither an I-ring nor NI. Let $K$ be a field and $D_{n}=K\left\{x_{n}\right\}$ with relation $x_{n}^{n+2}=0$, where $n$ is any non-negative integer and $K\left\{x_{n}\right\}$ is the free algebra generated by $x_{n}$ over $K$. Note $D_{n} \cong K[x] /\left(x^{n+2}\right)$, where $\left(x^{n+2}\right)$ is the ideal of $K[x]$ generated by $x^{n+2}$, with $K[x]$ the polynomial ring with an indeterminate $x$ over $K$. We use the ring in [18, Example 1.6]. Define $S_{n}=\left(\begin{array}{cc}D_{n} & D_{n} x_{n} \\ D_{n} x_{n} & D_{n}\end{array}\right)$. Notice that $J\left(S_{n}\right)=\left(\begin{array}{cc}D_{n} x_{n} & D_{n} x_{n} \\ D_{n} x_{n} & D_{n} x_{n}\end{array}\right)$ and $\frac{S_{n}}{J\left(S_{n}\right)} \cong\left(\begin{array}{cc}K & 0 \\ 0 & K\end{array}\right)$; hence $\left(\begin{array}{ll}f_{1} & f_{2}, \\ f_{3} & f_{4}\end{array}\right) \in S_{n}$ is invertible when the constant terms of $f_{1}$ and $f_{4}$ are both non-zero.

We first claim that $S_{n}$ is strongly AB. Notice that $I_{1}=K\left(\begin{array}{cc}x_{n}^{n+1} & 0 \\ 0 & 0\end{array}\right), I_{2}=$ $K\left(\begin{array}{cc}0 & x_{n}^{n+1} \\ 0 & 0\end{array}\right), I_{3}=K\left(\begin{array}{cc}0 & 0 \\ x_{n}^{n+1} & 0\end{array}\right)$ and $I_{4}=K\left(\begin{array}{cc}0 & 0 \\ 0 & x_{n}^{n+1}\end{array}\right)$ are non-zero ideals in $S_{n}$.

Take $X \subseteq S_{n}$ such that $r_{S_{n}}(X) \neq 0$. Then $X$ is contained in one of the following three kinds of subsets:

$$
\begin{aligned}
& Y_{1}=\left\{\left(\begin{array}{ll}
f_{1} & f_{2} \\
f_{3} & f_{4}
\end{array}\right) \in S_{n} \mid f_{i} \in D_{n} x_{n} \text { for } i=1,2,3,4\right\}, \\
& Y_{2}=\left\{\left(\begin{array}{ll}
f_{1} & f_{2} \\
f_{3} & f_{4}
\end{array}\right) \in S_{n} \mid f_{1} \in D_{n} \text { and } f_{i} \in D_{n} x_{n} \text { for } i=2,3,4\right\}, \\
& Y_{3}=\left\{\left(\begin{array}{ll}
f_{1} & f_{2} \\
f_{3} & f_{4}
\end{array}\right) \in S_{n} \mid f_{4} \in D_{n} \text { and } f_{i} \in D_{n} x_{n} \text { for } i=1,2,3\right\} .
\end{aligned}
$$


If $X \subseteq Y_{1}$, then $r_{S_{n}}(X)$ contains all $I_{j}$ 's; if $X \subseteq Y_{2}$, then $r_{S_{n}}(X)$ contains $I_{3}$ and $I_{4}$; if $X \subseteq Y_{3}$, then $r_{S_{n}}(X)$ contains $I_{1}$ and $I_{2}$. Consequently $S_{n}$ is strongly right $\mathrm{AB}$, and similarly we can conclude that $S_{n}$ is strongly left AB.

Next let $R=\prod_{i=0}^{\infty} S_{n}$. Then $R$ is strongly $\mathrm{AB}$ by Lemma 3.1(1), and so is every $S_{n}$.

Consider two sequences $\left(a_{n}\right),\left(b_{n}\right) \in R$ such that $a_{n}=\left(\begin{array}{cc}0 & x_{n} \\ 0 & 0\end{array}\right)$ and $b_{n}=\left(\begin{array}{cc}0 & 0 \\ x_{n} & 0\end{array}\right)$ for all $n$. Then $\left(a_{n}\right),\left(b_{n}\right) \in N(R)$, since $\left(a_{n}\right)^{2}=0=\left(b_{n}\right)^{2}$; but each component of $\left(a_{n}\right)+\left(b_{n}\right)$ is $\left(\begin{array}{cc}0 & x_{n} \\ x_{n} & 0\end{array}\right)$, and so $\left(a_{n}\right)+\left(b_{n}\right)$ is non-nilpotent. Thus $R$ is not NI.

The following lemma is due to Ramamurthi [29].

LeMma 3.10. (1) [29, Proposition 3.2] An IFP ring $R$ is strongly regular if and only if $R$ is right $S F$.

(2) $A$ ring $R$ is strongly regular if and only if $R$ is reduced and right SF if and only if $R$ is reduced and left $S F$.

Proof. (2) is proved by the left version of (1), [15, Theorem 3.2], [15, Corollary 1.13] and the fact that reduced rings are IFP.

Zhang and $\mathrm{Du}[\mathbf{3 8}$, Theorem 3] proved that a $\operatorname{ring} R$ is strongly regular if and only if $R$ is strongly right bounded and right SF. In the following theorems we give partial answers to Ramamurthi's question, extending the result of Zhang and Du and Lemma 3.10(1). Strongly regular rings are I-rings by Lemma 3.8(2) or [22, Proposition 9.4.1].

THEOREM 3.11. For a ring $R$ the following conditions are equivalent:

(1) $R$ is strongly regular;

(2) $R$ is strongly right $A B$ and right $S F$;

(3) $R$ is a strongly right $A B$ and left $S F$ I-ring.

Proof. (1) $\Rightarrow(2)$ and $(1) \Rightarrow(3)$ are obtained from [15, Theorem 3.2], [15, Corollary 1.13] and Lemma 3.8(2).

(2) $\Rightarrow(1)$ : Let $0 \neq a \in R$ with $a^{2}=0$. Then $r_{R}\left(\ell_{R}(a)\right)$ is non-zero right annihilator, and so there exists a non-zero ideal $I$ of $R$ such that $I \subseteq r_{R}\left(\ell_{R}(a)\right)$. So $\ell_{R}(a)=$ $\ell_{R}\left(r_{R}\left(\ell_{R}(a)\right)\right) \subseteq \ell_{R}(I)$. Here we claim that $\ell_{R}(I)=R$. Suppose not; then there exists a maximal right ideal $M$ containing $\ell_{R}(I)$. Since $R$ is right SF and $a \in M$, we can get $b \in M$ with $a=b a$ by the right version of [26, Proposition 5.4.3] (i.e. $R a \cap M=M a$ ). This implies that $1-b \in \ell_{R}(a) \subseteq \ell_{R}(I) \subseteq M$, and so $1 \in M$, a contradiction. Thus $\ell_{R}(I)=R$, and so $I=0$, which is also a contradiction, concluding that $R$ is reduced. Moreover $R$ is strongly regular by Lemma 3.10(1).

$(3) \Rightarrow(1)$ : First we have that $R / J(R)$ is strongly regular by Lemmas 3.8(3) and $3.10(1)$. Here we claim that $a R+r_{R}(a)=R$ for any element $a \in R$. Suppose not; then there exists a maximal right ideal $M$ containing $a R+r_{R}(a)$. But $M$ is also a maximal left ideal of $R$ by [15, Theorem 3.2], since $R / J(R)$ is strongly regular and $M \supseteq J(R)$. Now since $R$ is left SF and $a \in M$, there exists $b \in M$ such that $a=a b$ by [26, Proposition 5.4.3] (i.e. $a R \cap M=a M$ ). This implies that $1-b \in r_{R}(a) \subseteq M$, and so $1 \in M$, a contradiction. Thus $R$ is strongly regular from $a R+r_{R}(a)=R$.

In the following we get the same result as Theorem 3.11 with the NIness in place of the strong one-sided ABness. Strongly regular rings are reduced (hence NI) by [15, Theorem 3.2]. 
THEOREM 3.12. For a ring $R$, the following conditions are equivalent:

(1) $R$ is strongly regular;

(2) $R$ is right $S F$ and $N I$;

(3) $R$ is left $S F$ and NI.

Proof. It suffices to show $(2) \Rightarrow(1)$ and $(3) \Rightarrow(1)$ by [15, Corollary 1.13]. Let $R$ be a right SF and NI ring. Then $R / N(R)$ is reduced and right SF; hence $R / N(R)$ is strongly regular by Lemma 3.10(1). Since $J(R / N(R))=0$, we get $J(R) \subseteq N(R)$, entailing $J(R)=N(R)$. Then every maximal right or left ideal is two-sided because $R / J(R)$ is duo by [15, Theorem 3.2]. Here we will show $N(R)=0$. Assume on the contrary that there exists $0 \neq a \in N(R)$ such that $a^{2}=0$. Then there exists a maximal left ideal $M$ of $R$ containing $\ell_{R}(a)$. But $M$ is also a maximal right ideal of $R$ because $R / N(R)$ is duo, and so $R / M$ is a flat right $R$-module. Since $R$ is right SF, there exists $b \in M$ such that $a=b a$ by the right version of [26, Proposition 5.4.3]. Hence $1-b \in \ell_{R}(a) \subseteq M$, obtaining $1 \in M$, a contradiction. Consequently $N(R)=0$, and hence $R$ is strongly regular. The proof of $(3) \Rightarrow(1)$ is similar.

Recall that a ring $R$ is NI if and only if every minimal strongly prime ideal of $R$ is completely prime [18, Corollary 13]. Thus we can obtain the following from Theorem 3.12 and note that the condition ' $R$ is fully idempotent' is superfluous.

COROLlary 3.13. [37, Theorem 4] $A$ ring $R$ is strongly regular if and only if $R$ is a fully idempotent (i.e. every ideal is idempotent) right $S F$ ring such that every prime ideal is completely prime.

Strongly AB rings need not be abelian by Example 2.5(2), and abelain rings are also need not be strongly one-sided $\mathrm{AB}$ by the following.

EXAMPLE 3.14. Consider $R_{n}$ over $G F(2)$ as in the starting position of Section 2. Let $S_{n}=R_{2^{n-1}}$ for $n=1,2, \ldots$, and define a map $\sigma: S_{n} \rightarrow S_{n+1}$ by $A \mapsto\left(\begin{array}{ll}A & 0 \\ 0 & A\end{array}\right)$; then $S_{n}$ can be embedded into $S_{n+1}$ via $\sigma$ (i.e. $A=\sigma(A)$ in $S_{n+1}$ for $A \in S_{n}$ ). Then $\left\{S_{n}, \sigma_{n m}\right\}$, with $\sigma_{n m}=\sigma^{m-n}$ whenever $n \leq m$, is a direct system over $I=\{1,2, \ldots\}$. Set $R$ be the direct limit of $\left\{S_{n}, \sigma_{n m}\right\}$. Then $R=\bigcup_{n=1}^{\infty} S_{n}$. We first claim that $R$ is semiprime. Take $0 \neq A \in R$ such that $A$ is nilpotent. Then $A \in S_{n}$ for some $n$ such that the diagonal of $A$ is zero, and every non-zero entry of $A$ is 1 , say $A=\left(a_{s t}\right)$.

Let $i$ be the smallest such that the $i$ th row of $A$ contains a non-zero entry and $j$ be the smallest such that $a_{i j} \neq 0$ in the $i$ th row. Note that $i<j$ and $\left(i+2^{k}, j+2^{k}\right)$-entry of $A$ in $S_{k+1}$ is also 1 for $k=n, n+1, n+2, \ldots$. Use $e_{u v}$ to denote the square matrix in which $(u, v)$-entry is 1 and zero elsewhere. Let $A_{0}=A$ and $A_{1}=A_{0} B_{0} A_{0} \in A_{0} R A_{0}$, where $A_{0}$ is considered in $S_{n+1}$ and $B_{0}=e_{j\left(i+2^{n}\right)} \in S_{n+1}$. Say $A_{1}=\left(b_{s t}\right)$. Then $i$ is the smallest such that the $i$ th row of $A_{1}$ contains a non-zero entry and $j+2^{n}$ is the smallest such that $b_{i\left(j+2^{n}\right)} \neq 0$, actually $b_{i\left(j+2^{n}\right)}=1$, in the $i$ th row; hence $\left(i+2^{n+1}, j+2^{n}+2^{n+1}\right)$-entry of $A_{1}$ in $S_{n+2}$ is also 1 . Let $A_{2}=A_{1} B_{1} A_{1} \in A_{1} R A_{1}$, where $B_{1}=e_{\left(j+2^{n}\right)\left(i+2^{n+1}\right)} \in R_{n+2}$. Say $A_{2}=\left(c_{s t}\right)$. Then $i$ is the smallest such that the $i$ th row of $A_{2}$ contains a non-zero entry, and $j+2^{n}+2^{n+1}$ is the smallest such that $b_{i\left(j+2^{n}+2^{n+1}\right)} \neq 0$, actually $b_{i\left(j+2^{n}+2^{n+1}\right)}=1$, in the $i$ th row; hence $\left(i+2^{n+2}, j+2^{n}+2^{n+1}+2^{n+2}\right)$-entry of $A_{2}$ in $S_{n+3}$ is also 1 . Proceeding in this manner, we can show that the $\left(i, j+2^{n}+2^{n+1}+\cdots+2^{n+(k-1)}\right)$ entry of $A_{k}$ is also 1 for any $k$; hence we can obtain inductively a sequence $\left(A_{k}\right)_{k=0}^{\infty}$ each term of which is non-zero with $A_{k+1} \in A_{k} R A_{k}$. Thus $A$ is not strongly nilpotent and $A \notin P(R)$, concluding that $R$ is semiprime. 
Assume that $R$ is strongly one-sided AB. Then $R$ is reduced by Proposition 3.6, since $R$ is semiprime, a contradiction. Thus $R$ is neither strongly right nor strongly left AB.

While every $S_{n}$ is abelian and every non-zero idempotent in $S_{n}$ is such that the diagonal is 1 and zero elsewhere (i.e. the identity is the only non-zero idempotent), by [19, Lemma 2]. Thus $R$ is also abelian.

A ring $R$ is called directly finite if $x y=1$ implies $y x=1$ for $x, y \in R$. It is trivial to check that abelian rings are directly finite, whence the following proposition shows that directly finite rings unify the classes of strongly one-sided rings and abelian rings.

Proposition 3.15. Strongly one-sided AB rings are directly finite.

Proof. Let $R$ be a strongly right AB ring, and assume on the contrary that $x y=1$ but $y x \neq 1$ for some $x, y \in R$. Then $y x$ is a non-identity idempotent and $y x(1-y x)=0$ with $1-y x \neq 0$. Since $R$ is strongly right $\mathrm{AB}$, we have $y x R a=0$ for some non-zero $a \in R$; but $x R a=x y x R a=0$ implies $0 \neq a=x y a \in x R a=0$, a contradiction. Thus $R$ is directly finite. The proof for strongly left AB rings is similar.

Due to Anderson and Camillo [2], a ring $R$ satisfies $Z C_{2}$ if $a b=0$ implies $b a=0$ for $a, b \in R$. Rings satisfying $Z C_{2}$ are clearly IFP, but the converse need not hold by [23, Example 1.5].

COROllary 3.16. [2, Theorem 2.1] If a ring $R$ satisfies $Z C_{2}$, then $R$ is directly finite.

A one-sided Artinian ring $R$ is $\pi$-regular (i.e. for any $x \in R$ there are $y \in R$ and a positive integer $n$ such that $x^{n}=x^{n} y x^{n}$ ) by a simple computation.

LEMMA 3.17. Let $R$ be a strongly one-sided AB ring. If $R$ is one-sided Artinian, then $R / J(R)$ is a finite direct product of division rings, and especially each maximal one-sided ideal of $R$ is two-sided.

Proof. Let $R$ be one-sided Artinian. Then $R$ is an I-ring by [22, Proposition 9.4.1], and so $R / J(R)$ is reduced by Lemma 3.8(3). Thus $R / J(R)$ is a finite direct product of division rings, and it is immediate that each maximal one-sided ideal of $R$ is two-sided.

A quasi-Frobenius ring, introduced by Nakayama in 1939 [28], is defined to be right Artinian and right self-injective. A ring $R$ is quasi-Frobenius if and only if for each left ideal $I$ and right ideal $J$ in $R, \ell_{R}\left(r_{R}(I)\right)=I$ and $r_{R}\left(\ell_{R}(J)\right)=J[3$, Theorem 30.7].

Proposition 3.18. Let $R$ be a quasi-Frobenius ring. If $R$ is strongly one-sided $A B$, then $R$ is strongly bounded.

Proof. Since $R$ is right Artinian, every right ideal of $R$ contains a minimal right ideal. To prove that $R$ is strongly right bounded it suffices to show that every minimal right ideal is two-sided. Let $I$ be a minimal right ideal of $R$. Then since $R$ is quasiFrobenius, $J=\ell(I)$ is a maximal left ideal of $R$ with $I=r_{R}(J)$. But $J$ is two-sided by Lemma 3.17, and then we have $I=r_{R}(J)$ is two-sided. The proof for the left case is similar.

Denote the right (resp. left) socle - i.e. the intersection of all essential right (resp. left) ideals - of a ring $R$ by $\operatorname{Soc}\left(R_{R}\right)$ (resp. $\operatorname{Soc}\left({ }_{R} R\right)$ ). The simple right Noetherian domain in Example 2.3(5) is neither strongly right nor strongly left bounded. But 
reduced rings are strongly bounded when they satisfy descending chain condition (DCC) on essential right ideals as follows.

PROPOSITION 3.19. Let $R$ be a reduced ring satisfying DCC on essential right ideals. Then $R$ is a strongly bounded ring such that $R$ is a subdirect product of division rings, and every proper one-sided ideal consists of zero divisors.

Proof. We use freely the fact that reduced rings are non-singular, semiprime and abelian. Note that $\operatorname{Soc}\left(R_{R}\right)$ is itself an essential right ideal of $R$, since a finite intersection of essential submodules is also essential and $R$ satisfies DCC on essential right ideals.

Let $I$ be a non-zero right ideal of $R$. Then $\operatorname{Soc}\left(R_{R}\right) \cap I \neq 0$, and since $R$ is semiprime and abelian, $\operatorname{Soc}\left(R_{R}\right) \cap I$ contains a non-zero central idempotent. Thus $R$ is strongly right bounded.

To prove that $R$ is strongly left bounded it is enough to show that $\operatorname{Soc}\left({ }_{R} R\right)$ is essential left ideal of $R$, using a similar manner to the preceding one. First we get $\operatorname{Soc}\left({ }_{R} R\right)=\operatorname{Soc}\left(R_{R}\right)$, since $R$ is semiprime and abelian. Assume that $\operatorname{Soc}\left({ }_{R} R\right) \cap J=0$ for a left ideal $J$ of $R$. Then $\operatorname{Soc}\left({ }_{R} R\right) J=0$ and since $R$ is semiprime, we have $J \operatorname{Soc}\left({ }_{R} R\right)=0$; consequently $0=J \operatorname{Soc}\left({ }_{R} R\right)=J \operatorname{Soc}\left(R_{R}\right)$. But $R$ is right non-singular and $\operatorname{Soc}\left(R_{R}\right)$ is an essential right ideal of $R$, inducing $J=0$. Thus $R$ is strongly left bounded.

Every minimal prime ideal of $R$ is completely prime by [32, Proposition 1.11], since $R$ is reduced. So $R$ is a subdirect product of domains each of which satisfies DCC on essential right ideals by [5, Proposition 1.2(a)]. But a domain, satisfying DCC on essential right ideals, is a division ring by [5, Proposition 1.6]. Thus $R$ is a subdirect product of division rings. Every regular element of a semiprime ring is invertible by [5, Proposition 1.6] when it satisfies DCC on essential right ideals, and hence each proper right or left ideal of $R$ consists of zero divisors.

Note that the simple right Noetherian domain in Example 2.3(5) does not satisfy DCC on essential right ideals. Due to Yu [36], a ring is called right (resp. left) quasiduo if every maximal right (resp. left) ideal is two-sided. Right duo rings are clearly right quasi-duo, and it is straightforward that a right quasi-duo right primitive ring is a division ring. $\mathrm{Yu}$ [36, Lemma 2.3] showed that $R / J(R)$ is reduced for a right or left quasi-duo ring $R$. By Lemma 3.17, strongly one-sided AB rings are quasi-duo when they are one-sided Artinian. But quasi-duo rings need not be strongly onesided $\mathrm{AB}$ as can be seen by the $2 \times 2$ upper triangular matrix ring over a simple ring.

COROLlary 3.20. Let $R$ be a one-sided quasi-duo ring satisfying DCC on essential right ideals. Then $R / J(R)$ has the properties as in Proposition 3.19.

Proof. Since $R$ is one-sided quasi-duo and satisfies DCC on essential right ideals, $R / J(R)$ is a reduced ring satisfying DCC on essential right ideals by [36, Lemma 2.3] and [5, Proposition 1.2(a)]. Then Proposition 3.19 gives the result.

4. More examples of strongly one-sided $\mathbf{A B}$ rings. In this section we study some conditions and some ring extensions to extend the class of strongly one-sided $\mathrm{AB}$ rings. Let $R$ be a ring; $R[x]$ (resp. $R[[x]]$ ) denotes the polynomial ring (resp. power series ring) over $R$ with an indeterminate $x$ over $R$. 
The class of strongly one-sided $\mathrm{AB}$ rings is not closed under subrings by Example 3.2. In the following we see a kind of subring that inherits the strong ABness.

Proposition 4.1. Let $R$ be $a$ ring and $0 \neq e=e^{2} \in R$ such that eIe $\neq 0$ for any non-zero ideal I of $R$. If $R$ is strongly right $A B$, then so is eRe.

Proof. Let $R$ be a strongly right $\mathrm{AB}$ ring and $S=e R e$. Suppose that $r_{S}(X) \neq 0$ for $X \subseteq S$. Then since $R$ is strongly right AB there is a non-zero ideal $I$ of $R$ such that $I \subseteq r_{R}(X)$. Moreover $r_{S}(X)=r_{R}(X) \cap S \supseteq I \cap S=e I e$. By the condition eIe is a non-zero ideal of $S$, concluding that $S$ is strongly right $\mathrm{AB}$.

We next consider polynomial rings over a strongly right $\mathrm{AB}$ ring. By [17], there exists a strongly right bounded ring $R$ whose polynomial ring $R[x]$ is not strongly right bounded. By [20, Example 2], there exists an IFP ring $R$ whose polynomial ring $R[x]$ is not IFP. So it is natural to ask whether $R[x]$ is strongly right $\mathrm{AB}$ if a ring $R$ also is. We do not know the answer, but we find some conditions under which it is affirmative.

Proposition 4.2. Let $R$ be a ring and $J$ be an ideal of $R$ such that every element in $R \backslash J$ is regular and $J^{2}=0$. Then $R, R[x]$ and $R[[x]]$ are strongly $A B$.

Proof. Set $K=R \backslash J$. Let $A \subset R$ with $r_{R}(A) \neq 0$. Then $A \subseteq J$, and so $A J=0$; hence $R$ is strongly right AB. Similarly $R$ is strongly left AB.

Let $B \subset R[x]$ with $r_{R[x]}(B) \neq 0$, and suppose that $f(x) g(x)=0$ for $0 \neq f(x)=$ $\sum_{i=0}^{m} a_{i} x^{i} \in B$ and $0 \neq g(x)=\sum_{j=0}^{n} b_{j} x^{j} \in r_{R[x]}(B)$. We can let $a_{0}$ and $b_{0}$ be both nonzero and $m=n$. Since $R / J$ is a domain, [4, Lemma 1] implies that $a_{i} b_{j} \in J$ for all $i$ and $j ; a_{0} b_{0}=0$ gives $a_{0}, b_{0} \in J$. Assume $f(x) \notin J[x]$. Then $g(x) \in J[x]$ because each $a_{i} b_{j}$ is in $J$. Say that $k$ is smallest such that $a_{k} \in K$. Then $k \geq 1$. From $J^{2}=0$ we get $0=a_{0} b_{k}+a_{1} b_{k-1}+\cdots+a_{k-1} b_{1}+a_{k} b_{0}=a_{k} b_{0}$, entailing $b_{0}=0$, a contradiction. Thus $f(x) \in J[x]$, and we get $B \subset J[x]$. Consider the non-zero ideal $J[x]$ of $R[x]$. From $J[x]^{2}=0$ we have $B J[x]=0$, concluding that $R[x]$ is strongly right AB. Similarly $R[x]$ is strongly left $\mathrm{AB}$. The proof for $R[[x]]$ is similar.

Given a ring $R$ and a bimodule ${ }_{R} M_{R}$, the trivial extension of $R$ by $M$, denoted by $T(R, M)$, is the ring $R \oplus M$ with the usual addition and the multiplication $\left(r_{1}, m_{1}\right)\left(r_{2}, m_{2}\right)=\left(r_{1} r_{2}, r_{1} m_{2}+m_{1} r_{2}\right)$. This is isomorphic to the ring of all matrices $\left(\begin{array}{ll}r & m \\ 0 & r\end{array}\right)$, where $r \in R$ and $m \in M$ and the usual matrix operations are used.

Let $R$ be a commutative ring and $h$ be a ring endomorphism of $R$. For an $R$-module $M$, the multiplication $(a, m)(b, n)=(a b, h(a) n+b m)$ gives a ring structure to $R \oplus M$, denoted by $R(+)_{h} M$.

Corollary 4.3. (1) Let $R$ be a commutative domain and $M$ be an R-module. If $M$ is torsion-free, then $T(R, M)$ and $T(R, M) x]$ are both strongly $A B$.

(2) Let $K$ be a field, $h$ be a non-zero ring endomorphism of $K$ and $V$ be a $K$-vector space. Then $K(+)_{h} V$ and $\left(K(+)_{h} V\right)[x]$ are both strongly $A B$.

(3) Let $R$ be a local ring with $J(R)^{2}=0$. Then $R$ and $R[x]$ are both strongly $A B$.

Proof. (1) Let $J=\{(a, m) \in T(R, M) \mid a=0\}$; then $J^{2}=0$ and $R \backslash J$ is the set of all regular elements in $T(R, M)$. (2) Let $J=\left\{(k, v) \in K(+)_{h} V \mid k=0\right\}$; then $J^{2}=0$ and $R \backslash J$ is the set of all regular elements in $K(+)_{h} V$. (3) Let $R$ be a local ring. Then $R \backslash J(R)$ is clearly the set of all regular elements in $R$. Thus we get the results from Proposition 4.2. 
The following is similar to Proposition 4.2.

PROPOSITION 4.4. If $R$ is a local ring with nilpotent $J(R)$, then $R$ and $R[[x]]$ are strongly $A B$.

Proof. Let $R$ be a local ring such that $J(R)^{k}=0$ and $J(R)^{k-1} \neq 0$. Then $R$ is strongly $\mathrm{AB}$ by Lemma $3.4(6)$, and $\frac{R[[x]]}{J(R)[[x]]} \cong \frac{R}{J(R)}[[x]]$ is a domain such that $(J(R)[[x]])^{k-1}$ is a non-zero nilpotent ideal of $R[[x]]$. Let $A \subset R[[x]]$ with $r_{R[[x]]}(A) \neq 0$, say $f(x) g(x)=0$ for $0 \neq f(x) \in B$ and $0 \neq g(x) \in r_{R[x]}(B)$. Here we can suppose that $a_{0}$ and $b_{0}$ are both non-zero. Put $T=\frac{R[[x]]}{J(R)[[x]]}$, and use $h(x)$ in place of $h(x)+J(R)[[x]]$ for simplicity. Assume $f(x) \notin J(R)[[x]]$. Then $f(x)=x^{n} f_{0}(x)=f_{0}(x) x^{n}$ in $T$ for some nonnegative integer $n$ and $f_{0}(x) \in T$ with invertible constant term. So $f_{0}(x)$ is invertible, say $f_{0}(x) h(x)=h(x) f_{0}(x)=1$; hence $h(x) f_{0}(x) x^{n}=x^{n}$ and $\left(1-h(x) f_{0}(x)\right) x^{n} \in J(R)[[x]]$. But $J(R)[[x]]^{k}=0$, and so we get $\left(1-h_{0}(x) f_{0}(x)\right) x^{n k}=0$ (hence $\left.1-h_{0}(x) f_{0}(x)=0\right)$ for some $h_{0}(x) \in R[[x]]$. Now we have $0=h_{0}(x) f(x) g(x)=h_{0}(x) f_{0}(x) g(x) x^{n}=g(x) x^{n}$, and so $g(x)=0$, a contradiction. Thus $f(x) \in J(R)[[x]]$ and $A(J(R)[[x]])^{k-1}=0$, entailing that $R[[x]]$ is strongly right $\mathrm{AB}$. Similarly $R[[x]]$ is strongly left $\mathrm{AB}$.

Consider $R_{n}$ as in Section 2 over a a local ring with nilpotent Jacobson radical. Then $R_{n}$ and $R_{n}[[x]]$ are both strongly $\mathrm{AB}$ by Theorem 2.2, Lemma 3.4(6) and Proposition 4.4.

PROPOSITION 4.5. Let $R$ be a ring and $n$ any positive integer. Then $R$ is strongly right $A B$ if and only if so is $R[x] /\left(x^{n}\right)$, where $\left(x^{n}\right)$ is the ideal of $R[x]$ generated by $x^{n}$.

Proof. Put $S=R[x] /\left(x^{n}\right)$, and use $h(x)$ in place of $h(x)+\left(x^{n}\right)$ for simplicity. Suppose that $R$ is strongly right AB. Let $A \subset S$ with $r_{S}(A) \neq 0$, and take non-zero $g(x)=\sum_{j=0}^{n} b_{j} x^{j}$ in $r_{S}(A)$. Here we can assume $b_{0} \neq 0$. Consider the subset $B=\{a \in$ $R \mid a=a_{m}$ for $\left.0 \neq f(x)=\sum_{i=m}^{n} a_{i} x^{i} \in A\right\}$ of $R$. Then $r_{R}(B) \neq 0$, since $b_{0} \in r_{R}(B)$. But $R$ is strongly right $\mathrm{AB}$, and so there is a non-zero ideal of $R$ such that $I \subseteq r_{R}(B)$. Now we have $A(I[x])^{n-1}=0$, concluding that $S$ is strongly right $\mathrm{AB}$.

Conversely suppose that $S$ is strongly right $\mathrm{AB}$. Let $A \subseteq R$ with $r_{R}(A) \neq 0$. Note that $r_{R}(A)=r_{S}(A) \cap R$. Since $r_{R}(A) \neq 0$, we get $r_{S}(A) \neq 0$. But $S$ is strongly right $\mathrm{AB}$, so there is a non-zero ideal $L$ of $S$ such that $r_{S}(A) \supseteq L$. Let $L_{0}$ be the set of all coefficients of all polynomials in $L$. Then clearly $L_{0}$ is a non-zero ideal of $R$. Now $A L_{0}=0$, and so $R$ is strongly right $\mathrm{AB}$.

Due to Rege and Chhawchharia [31], a ring $R$ is called Armendariz if $a_{i} b_{j}=0$ for all $i$ and $j$ whenever polynomials $f(x)=\sum_{i=0}^{m} a_{i} x^{i}$ and $g(x)=\sum_{j=0}^{n} b_{j} x^{j}$ in $R[x]$ satisfy $f(x) g(x)=0$. Reduced rings are Armendariz by [4, Lemma 1], and Armendariz rings are abelian by the proof of [1, Theorem 6]. Commutative (hence strongly AB) rings need not be Armendariz by [31, Example 3.2].

Proposition 4.6. Let $R$ be an Armendariz ring. Then $R$ is strongly right $A B$ if and only if so is $R[x]$.

Proof. Suppose that $R$ is strongly right AB. Let $A \subseteq R[x]$ with $r_{R[x]}(A) \neq 0$ and $B$ be the set of all coefficients of polynomials in $A$. Take non-zero $f(x)=a_{0}+a_{1} x+\cdots+$ $a_{n} x^{n}$ in $r_{R[x]}(A)$. Then for any $g(x)=b_{0}+b_{1} x+\cdots+b_{m} x^{m} \in A, g(x) f(x)=0$. Since $R$ is Armendariz, $b_{i} a_{j}=0$ for all $i, j$. Thus $a_{j} \in r_{R}(B)$ for any $j=1,2, \ldots, n$, entailing $r_{R}(Y) \neq 0$. Since $R$ is strongly right $\mathrm{AB}$, there exists a non-zero ideal $I$ of $R$ such that 
$r_{R}(B) \supseteq I$. Then $I[x]$ is a non-zero ideal of $R[x]$ such that $r_{R[x]}(A) \supseteq I[x]$. Thus $R[x]$ is strongly right $\mathrm{AB}$. The proof of the converse is similar to the second part of the proof of Proposition 4.5.

Proposition 4.7. Let $R$ be a ring and $\Delta$ be a multiplicatively closed subset of $R$ consisting of central regular elements. Then $R$ is strongly right $A B$ if and only if so is $\Delta^{-1} R$.

Proof. Suppose that $R$ is strongly right $\mathrm{AB}$, and let $A \subseteq \Delta^{-1} R$ such that $r_{\Delta^{-1} R}(A) \neq$ 0 . Let $B=\left\{a \in R \mid u^{-1} a \in A\right\} \subseteq R$.

Take $0 \neq v^{-1} b \in r_{\Delta^{-1} R}(A)$; then $B b=0$, and so $r_{R}(B) \neq 0$. Since $R$ is strongly right $\mathrm{AB}$, there exists a non-zero ideal $I$ of $R$ such that $r_{R}(B) \supseteq I$. Then for any $u^{-1} a \in A$, $u^{-1} a I=0$, since $a \in B$ and $A I=0$. Note that $\Delta^{-1} I$ is a non-zero ideal of $\Delta^{-1} R$. Since $I \subseteq r_{\Delta^{-1} R}(A)$, we have $\Delta^{-1} I \subseteq r_{\Delta^{-1} R}(X)$. Thus $\Delta^{-1} R$ is strongly right $\mathrm{AB}$.

Conversely, suppose that $\Delta^{-1} R$ is strongly right $\mathrm{AB}$, and let $A \subseteq R$ such that $r_{R}(A) \neq 0$. Then $r_{\Delta^{-1} R}(A) \neq 0$. Since $\Delta^{-1} R$ is strongly right $\mathrm{AB}$, there exists a nonzero ideal $L$ of $\Delta^{-1} R$ such that $r_{\Delta^{-1} R}(A) \supseteq L$. Take $0 \neq v^{-1} b \in L$. Since $v^{-1}$ is central, we have $v^{-1} R b R=R v^{-1} b R \subseteq L$. Thus $A v^{-1} R b R=v^{-1} A R b R=0$, and so $A R b R=0$. Thus $R$ is strongly right $\mathrm{AB}$.

The ring of Laurent polynomials in $x$, coefficients in a ring $R$, consists of all formal sums $\sum_{i=k}^{n} m_{i} x^{i}$ with obvious addition and multiplication, where $m_{i} \in R$ and $k, n$ are (possibly negative) integers; denote it by $R\left[x ; x^{-1}\right]$.

COROLlary 4.8. Let $R$ be an Armendariz ring. Then the following conditions are equivalent:

(1) $R$ is strongly right $A B$;

(2) $R[x]$ is strongly right $A B$;

(3) $R\left[x ; x^{-1}\right]$ is strongly right $A B$.

Proof. $(1) \Leftrightarrow(2)$ is Proposition 4.6. (2) $\Leftrightarrow(3)$ : Let $\Delta=\left\{1, x, x^{2}, \ldots\right\}$. Then $\Delta$ is a multiplicatively closed subset of $R[x]$ consisting of central regular elements. Note that $R\left[x ; x^{-1}\right]=\Delta^{-1} R[x]$. So the equivalence is obtained from Proposition 4.7.

Let $R, S$ be rings and $f: R \rightarrow S$ be an isomorphism. $R \oplus S$, denoted by $R(+)_{f} S$, is a ring with the usual addition and the multiplication $\left(r_{1}, s_{1}\right)\left(r_{2}, s_{2}\right)=\left(r_{1} r_{2}, f\left(r_{1}\right) s_{2}+\right.$ $\left.s_{1} f\left(r_{2}\right)\right)$.

Proposition 4.9. Let $R, S$ be rings and $f: R \rightarrow S$ be an isomorphism. Then $R$ is strongly right $A B$ if and only if so is $R(+)_{f} S$.

Proof. Apply the proof of Theorem 2.2.

AcKNOWLEDEGEMENTS. The second author was supported by the Korea Research Foundation Grant (KRF-2006-521-C00003) funded by the Korean Government (MOEHRD), and the third author was supported for two years by Busan National University Research Grant.

\section{REFERENCES}

1. D. D. Anderson and V. Camillo, Armendariz rings and Gaussian rings, Comm. Algebra 26 (1998), 2265-2272. 
2. D. D. Anderson and V. Camilo, Semigroups and rings whose zero products commute, Comm. Algebra 27 (1999), 2847-2852.

3. F. W. Anderson and K. R. Fuller, Rings and categories of modules (Springer-Verlag, New York, 1992).

4. E. P. Armendariz, A note on extensions of Baer and P.P.-rings, J. Austral. Math. Soc. 18 (1974), 470-473.

5. E. P. Armendariz, Rings with DCC on essential left ideals, Comm. Algebra 8 (1980), 299-308.

6. H. E. Bell, Near-rings in which each element is a power of itself, Bull. Austral. Math. Soc. 2 (1970), 363-368.

7. G. F. Birkenmeier and R. P. Tucci, Homomorphic images and the singular ideal of a strongly right bounded ring, Comm. Algebra 16 (1988), 1099-1122.

8. A. W. Chatters and W. Xue, On right duo p.p. rings, Glasgow Math. J. 32 (1990), 221-225. 1127.

9. J. Chen, On von Neumann regular rings and SF-rings, Math. Japon. 36 (1991), 1123-

10. R. C. Courter, Finite dimensional right duo algebras are duo, Proc. Am. Math. Soc. 84 (1982), 157-161.

11. L. M. de Narbonne, Anneaux semi-commutatifs et unis riels anneaux dont les id aux principaux sont idempotents. In Proceedings of the 106th National Congress of Learned Societies, (Bibliotheque Nationale, Paris, 1982), 71-73.

12. K. E. Eldridge, Orders for finite noncommutative rings with unity, Am. Math. Monthly 73 (1966), 376-377.

13. C. Faith, Algebra II ring theory (Springer-Verlag, Berlin, 1976).

14. E. H. Feller, Properties of primary noncommutative rings, Trans. Am. Math. Soc., 89 (1958), 79-91.

15. K. R. Goodearl, Von Neumann regular rings (Pitman, London, 1979).

16. Y. Hirano, On rings whose simple modules are flat, Can. Math. Bull. 37 (1994), 361-364.

17. Y. Hirano, C. Y. Hong, J. Y. Kim and J. K. Park, On strongly bounded rings and duo rings, Comm. Algebra 23 (1995), 2199-2214.

18. C. Y. Hong and T. K. Kwak, On minimal strongly prime ideals, Comm. Algebra 28 (2000), 4867-4878.

19. C. Huh, H. K. Kim and Y. Lee, P.p. rings and generalized p.p. rings, J. Pure Appl. Algebra 167 (2002), 37-52.

20. C. Huh, Y. Lee and A. Smoktunowicz, Armendariz rings and semicommutative rings, Comm. Algebra 30 (2002), 751-761. 1943).

21. N. Jacobson, The theory of rings (American Mathematical Society, Providence, RI,

22. N. Jacobson, Structure of rings, 2nd ed., Colloquium Publication 37 (American Mathematical Society, 1964).

23. N. K. Kim and Y. Lee, Extensions of reversible rings, J. Pure Appl. Algebra 185 (2003), $207-223$.

24. G. Köthe, Die Struktur der Ringe deren Restklassenring nach dem Radikal vollständig reduzibel ist, Math. Z., 42 (1930), 161-186.

25. R. Kruse and D. Price, Nilpotent rings (Gordon and Breach, New York, 1969). 1966).

26. J. Lambek, Lectures on rings and modules (Blaisdell, Waltham, MA/Toronto/London,

27. G. Marks, On 2-primal Ore extensions, Comm. Algebra 29 (2001), 2113-2123.

28. T. Nakayama, On Frobeniusean algebras. I. Ann. Math. (2) 40 (1939), 611-633.

29. V. S. Ramamurthi, On the injectivity and flatness of certain cyclic modules, Proc. Am. Math. Soc. 48 (1975), 21-25.

30. M. B. Rege, On von Neumann regular rings and SF-rings, Math. Japon. 31 (1986), 927-936.

31. M. B. Rege and S. Chhawchharia, Armendariz rings, Proc. Jpn. Acad. Ser. A Math. Sci. 73 (1997), 14-17.

32. G. Shin, Prime ideals and sheaf representation of a pseudo symmetric ring, Trans. Am. Math. Soc. 84 (1973), 43-60.

33. Y. Xiao, SF-rings and exellent extensions, Comm. Algebra 22 (1994), 2463-2471. 
34. W. Xue, On strongly right bounded finite rings, Bull. Austral. Math. Soc. 44 (1991), $353-355$.

35. W. Xue, Structure of minimal noncommutative duo rings and minimal strongly bounded non-duo rings, Comm. Algebra 20 (1992), 2777-2788.

36. H.-P. Yu, On quasi-duo rings, Glasgow Math. J. 37 (1995), 21-31. 128.

37. R. Yue Chi Ming, Maximal ideals in regular rings, Hokkaido Math. J. 12 (1988), 119-

38. J. Zhang and X. Du, Von Neumann regularity of SF-rings, Comm. Algebra 21 (1993), 2445-2451. 